\title{
Screening with the Pap test
}

\section{Deaths from invasive cervical cancer occur mostly among women who do not undergo regular screening}

The rate of death from cervical cancer is reduced by more than $80 \%$ among women who have regular Papanicolaou (Pap) screening. ${ }^{1}$ Women of lower socioeconomic status and those who are older, First Nations or immigrants are less likely to be screened regularly. ${ }^{1}$ Screening intervals shorter than three years increase the risk of finding and investigating abnormalities that mostly resolve spontaneously. ${ }^{2,3}$ Recommended intervals vary by jurisdiction, but longer intervals require organized screening and recall programs to maintain high participation rates.

Testing for human papillomavirus (HPV) infection has a developing role in screening for cervical cancer

Many laboratories routinely test for HPV on Pap samples that have specific cellular abnormalities. Primary HPV testing (i.e., without cytology) is currently not offered in Canada. The evidence for this strategy is still being developed and may change practice in the near future. A recent meta-analysis of randomized controlled trials found a small absolute reduction in invasive cancer with primary HPV testing, but it is too early to assess mortality. ${ }^{5}$

\section{Some women do not require Pap screening}

Ages at which Pap screening should start vary by jurisdiction, but most guidelines agree that harm from false-positive results outweighs potential benefits of Pap screening in young women. ${ }^{4}$ Women who have had a total hysterectomy for a benign disorder and women over 70 years of age who have had three normal test results within 10 years do not require Pap screening. ${ }^{4}$
Women vaccinated against HPV infection still require Pap screening

HPV types 16 and 18 are present in about $70 \%$ of cervical cancers worldwide and are targeted in HPV vaccines. However, over 40 other types also colonize the genital tract, some of which are associated with cancer. ${ }^{6}$ Because most cervical cancers occur in women over 35 years of age, longer follow-up of HPV vaccine trials are required to measure their effectiveness. ${ }^{2}$

See references, Appendix 1, www.cmaj.ca /lookup/suppl/doi:10.1503/cmaj.141199/-/DC1

Resources for physicians and patients on cervical cancer screening are available on the Canadian Task Force on Preventive Health Care website (http://canadiantaskforce.ca/ctfphc -guidelines/2013-cervical-cancer/).
CMAJ is collaborating with Choosing Wisely Canada (www.choosingwiselycanada.org), with support from Health Canada, to publish a series of articles describing how to apply the Choosing Wisely Canada recommendations in clinical practice.
Box: Choosing Wisely Canada recommendations on Pap screening ${ }^{4}$

- Don't use the Pap test for screening in women who are under 21 or more than 69 years of age*

- Don't do Pap screening annually in women with previously normal results

- Don't do Pap tests in women who have had a full hysterectomy for a benign disorder

*Screening should stop at age 70 if the results of three previous tests were normal.
Adding bimanual pelvic examination to Pap screening does not reduce the risk of disease

Routine screening with bimanual pelvic examination does not reduce the risk of disease, whether cervical, uterine or ovarian. ${ }^{7}$ This examination is uncomfortable for many women and makes Pap screening less acceptable., ${ }^{7,8}$
Competing interests: James Dickinson is a member of the Canadian Task Force on Preventive Health Care, which is a voluntary, unpaid position supported by a grant that provides reimbursement of expenses for meetings. No competing interests were declared by David Ponka.

This article has been peer reviewed.

Affiliations: Department of Family Medicine (Ponka), University of Ottawa, Ottawa, Ont.; Departments of Family Medicine and Community Health Sciences (Dickinson), University of Calgary, Calgary, Alta.

Correspondence to: David Ponka, dponka @ bruyere.org

CMAJ 2014. DOI:10.1503/cmaj.141199 